\title{
Assessment of Indications, Co-Morbidities and Complications in Patients Pre- and Post-Tonsillectomy and Adenoidectomy - A Retrospective Study
}

\author{
Subash Chendrashekar ${ }^{1}$, Rishabh Jain² ${ }^{2}$ Arif Mohammed Sagar K.P. ${ }^{3}$ \\ 1,2,3 Department of Otolaryngology, Mysore Medical College and Research Institute, Mysore, Karnataka, India.
}

\section{ABSTRACT}

\section{BACKGROUND}

Tonsillectomy and adenoidectomy are the common procedures done by otolaryngologists all over the world. The purpose of this study is to evaluate the indications, complications and post-operative recovery in uncomplicated patients undergoing tonsillectomy and adenoidectomy.

\section{METHODS}

A retrospective study was done in Mysore Medical College from May 2019 to May 2020 in patients attending ENT outpatient department who underwent tonsillectomy or adenoidectomy or both.

\section{RESULTS}

A total of 283 patients were selected for the study who underwent the procedure during the study period. Obstruction was the main indication for the procedure in the age group of 2 - 4 years accounting for 32 out of 37 cases (86.4\%), 107 out of 183 (58.4\%) in the age group of 4 - 10 years and 25 out of 63 cases (39.6\%) in the age group of 10 - 18 years. While infection accounted for highest number of cases in older age group, i.e., 38 out of 63 cases (60.4\%) in the age group of 10 - 18 years, 76 out 183 cases ( $41.6 \%)$ in the age group of $4-10$ years and only 5 out of 37 cases (13.6 $\%)$ in younger age group of 2 - 4 years. Obesity is the most common co-morbidity (22.9\%) followed by otitis media with effusion (15.9\%) and allergic rhinitis (7.7\%). Most common complication was fever (26.8\%) followed by delay in oral intake $(22.2$ $\%)$ and pain (16.6\%). All the patients recovered within 48 hours and were discharged.

\section{CONCLUSIONS}

Adenoidectomy and tonsillectomy are safe, effective procedures. Obstruction is the major indication in younger age group which has been on the rise in recent few decades as opposed to infection which is the major indication in older age group children. Co-morbidity observed in the patients was obesity. Complications were easily detected and treated.

\section{KEY WORDS}

Tonsillectomy, Adenoidectomy, Obstruction, Infection

\author{
Corresponding Author: \\ Dr. Rishabh Jain, \\ 11, Naval Vihar, Baghpat Road, \\ Meerut, Uttar Pradesh, India. \\ E-mail: dr.rishabhjain19@gmail.com
}

DOI: $10.14260 / \mathrm{jemds} / 2021 / 47$

How to Cite This Article:

Chendrashekar S, Jain R, Sagar K.P.A.M. Assessment of indications, co-morbidities and complications in patients pre- and posttonsillectomy and adenoidectomy - a retrospective study. J Evolution Med Dent Sci 2021;10(04):217-221, DOI: $10.14260 /$ jemds/2021/47

Submission 23-09-2020,

Peer Review 27-11-2020,

Acceptance 04-12-2020,

Published 25-01-2021.

Copyright (c) 2021 Subash Chendrashekar et al. This is an open access article distributed under Creative Commons Attribution License [Attribution 4.0 International (CC BY 4.0)] 


\section{BACKGROUND}

Tonsillectomy and adenoidectomy were developed hundreds of years ago and are probably the most common procedures performed by otolaryngologist all over the world. Tonsillectomy was first described in the Hindu writings dating $1000 \mathrm{BC}$ depicting a partial tonsillectomy using fingernail dissection. Total tonsillectomy was described by Cornelius Celsius in the first century AD using a fingernail and metal hook followed by vinegar irrigation for haemostasis. Snare tonsillectomy was described by Galen whose technique was employed for approximately 400 years. Philip Syng Physick developed the tonsillotome in 1828 based on a French guillotine and served as a basis for subsequent modification. Modern cold steel was popularised by British and American otolaryngologists in the early $20^{\text {th }}$ century. Tonsillectomy is a procedure of antiquity, and date as far back as the first century A.D. ${ }^{1}$ It contributes to the improvement in the quality of life and at times can help with airway difficulties when performed for major indications like obstruction and infection of upper respiratory tract. ${ }^{2}$ These procedures have showed benefits in $70-80 \%$ consistently and these procedures are found to be curative and follow up surgeries are not required.

Worldwide, the most common indications for performing these procedures have been classified into two main categories namely, obstruction and infection. For a very long time until 1980s, infection was considered as the main indication for (adeno) tonsillectomy; however, recently, the main indication reported for the procedures is obstruction mainly for adenoidectomy in younger age group. These procedures gained wide popularity as a preventive measure of Group A beta-haemolytic streptococcal (GABHS) pharyngotonsillitis and the complication associated with itrheumatic heart disease. During this era, the children who didn't have any history of streptococcal infection became candidates of the surgery because another family member suffered from frequent streptococcal disease or rheumatic heart disease. With the advent of potent antibiotics, specifically penicillin, the incidence of these procedures being performed for infection came down and following that these procedures were mainly performed for any obstructive pathology.

Recent studies show obstruction as the primary indication as opposed to infection being the major indication for a long time. This can be due to introduction of antibiotics which decreased the infection rates as claimed by the proponents of the view. ${ }^{3}$ An audit refuted the claim by The Mckinsey National Health Service which stated that tonsillectomy was a relatively ineffective and often an unjustified procedure. ${ }^{4}$

In most developing countries the assessment is essentially clinical with detailed history taking and physical examination due to the lack of sleep laboratories required to do polysomnography, the gold standard for diagnosing obstructive sleep apnoea syndrome (OSAS).

Various techniques have been proposed to be highly effective, safe and with rapid recovery. The techniques available to surgeons include convention cold steel, electrocautery, microdebrider, coblation, laser and radio frequency. ${ }^{5}$ The basis of development of these techniques are the intra-operative factors (blood loss, time taken for surgery), postoperative results (pain, bleeding, dehydration, time taken for complete healing), and other complications like vomiting and hospitalisation time for different surgical techniques for the procedures. Microdebrider intracapsular tonsillectomy is associated with lower mortality and morbidity as compared to cold steel, coblation, electro dissection, laser and radio frequency. Our centre uses the traditional cold knife dissection and adenoid curettage for tonsillectomy and adenoidectomy respectively.

\section{METHODS}

In this retrospective study, the case notes of 283 patients were retrieved from a total of 302 patients who underwent tonsillectomy and adenoidectomy (T\&A) from May 2019 to May 2020 done by the qualified otolaryngologist at the department of E.N.T., Mysore Medical College and Research Institute according to the criteria laid down by the American Academy of Otolaryngology, Head and Neck Surgery [Table 1] after taking informed consent from the patients and clearance from the ethical committee.

Pre-operative analysis included variables such as age, gender, symptoms, past medical history, past surgical history, physical examination, X-rays of the nasopharynx and routine blood investigations.

All the case sheets retrieved were divided into three age ranges: 0 - 3 years, 4 - 10 years and 11 - 18 years of age. The indications were divided into two main categories being infections which included rhinosinusitis, rhinopharyngitis, pharyngotonsillitis, otitis media secondary to recurrent adenoiditis and peritonsillar abscess. The second main indication was obstruction which comprised of tonsil and / or adenoid hypertrophy upper airway obstruction and / or obstructive sleep apnoea (OSA) / sleep-disordered breathing (SDB). SDB / OSA was diagnosed purely on the basis of history taking and clinical assessment due to the unavailability of sleep laboratories for polysomnography in our centre.

Any per-op upper respiratory tract infection (URTI) was treated before the procedures. All the procedures were done under general anaesthesia. Adenoidectomy was performed first with curettage technique. Obstructive adenoids were removed with three or four strokes and bleeding was controlled by packing the nasopharynx with adenoid pack. Tonsillectomy was done following adenoidectomy using cold knife dissection technique and bleeding was controlled by ligation of the bleeders in the tonsillar fossa. Tonsillar bed and nasopharynx were rechecked for any bleeding after completion of both the procedures and before shifting the patient to the wards in the post anaesthetic room where the patients was kept for 3 hours post op for observation.

Concurrent procedures were done like myringotomy with grommet insertion and myringotomy alone for patients with otitis media with effusion.

Post-op variables were studied which included the duration of hospital stay. Time to first significant oral intake, vomiting, haemorrhage and other complication occurring during the recovery period.

\section{Exclusion Criteria}

Syndromic patients with congenital anomalies, neoplastic cases, facial defects and patients with incomplete case records. 


\begin{tabular}{|c|c|}
\hline Criterion & Definition \\
\hline $\begin{array}{l}\text { Minimum frequency of sore throat } \\
\text { episodes }\end{array}$ & $\begin{array}{c}7 \text { or more episodes in the preceding year } \\
5 \text { or more episode in each of the preceding } 2 \text { years } \\
3 \text { or more episode in each of the preceding } 3 \text { years }\end{array}$ \\
\hline $\begin{array}{l}\text { Clinical features (sore throat+ one or } \\
\text { more qualifies as counting episode) }\end{array}$ & $\begin{array}{c}\text { Temperature }>38.3 \text { degree Celsius }(>101 \mathrm{~F} \text { ), cervical lymphadenopathy (tender lymph node or }>2 \mathrm{~cm} \text { ) tonsillar exudate positive culture } \\
\text { for group A beta haemolytic streptococcus }\end{array}$ \\
\hline $\begin{array}{l}\text { Hypertrophy causing upper airway } \\
\text { obstruction (sleep apnoea) }\end{array}$ & $\begin{array}{c}\text { Severe dysphagia (trouble swallowing), sleep disorder, or cardiopulmonary complications. usually, removal of both tonsils and adenoids are } \\
\text { indicated }\end{array}$ \\
\hline Peritonsillar abscess & Unresponsive to medical management and drainage documented by surgeon, unless surgery performed during acute stage \\
\hline Streptococcal carrier & Chronic or recurrent tonsillitis associated with the streptococcal carrier state and not responding to beta lactamase resistant antibiotics \\
\hline Unilateral enlargement & $\begin{array}{l}\text { Unilateral tonsil hypertrophy presumed neoplastic. Although without other indications (abnormal appearance, physical examination, } \\
\text { symptoms or history) most asymmetries can be followed conservatively }\end{array}$ \\
\hline Adenoidectomy done & $\begin{array}{l}\text { Recurrent acute otitis media or chronic serous otitis media. Adenoidectomy should not be performed with the insertion of the first set of } \\
\text { myringotomies (ear) tubes unless there is another indication for adenoidectomy besides chronic otitis media. However, repeat surgery for } \\
\text { chronic otitis media should consist of adenoidectomy with myringotomy (with or without myringotomy (ear) tube replacement) }\end{array}$ \\
\hline & Table 1. Criteria for Tonsillectomy \\
\hline
\end{tabular}

\section{RESULTS}

A total of 283 files were reviewed from a total of 303 selected cases who underwent $\mathrm{T} \& \mathrm{~A}$ due to the lack of complete case records. There were 175 males (61.8\%) and 108 females $(38.2$ $\%)$. Their age ranged from 2 years and 8 months to 18 years and were divided into three categories: 2 to 4 years, 4 to 10 years and 10 to 18 years. A total of 164 cases (57.9\%) were operated for obstruction as primary indication and 119 (42.1 $\%$ ) cases were operated for infection as the primary indication. Of the group of people having both the indication for procedures, only the primary indication was considered to segregate patients into the two major groups. As observed in Table 2, obstruction was the main indication for the procedure in the age group 2 - 4 years accounting for 32 out of 37 cases $(86.4 \%), 107$ out of $183(58.4 \%)$ in the age group of $4-10$ years and 25 out of 63 cases $(39.6 \%)$ in the age group of 10 18 years. While infection accounted for higher cases in older age group i.e. 38 out of 63 cases (60.4\%) in the age group of 10 - 18 years, 76 out 183 cases (41.6\%) in the age group of 4 10 years and only 5 out of 37 cases (13.6\%) in younger age group of 2 - 4 years.

\begin{tabular}{|cccc|}
\hline Age Group (Years) & Obstruction & Infection & Total \\
$2-4$ & $32[86.4 \%]$ & $5[13.6 \%]$ & 37 \\
$4-10$ & $107[58.4 \%]$ & $76[41.6 \%]$ & 183 \\
$10-18$ & $25[39.6 \%]$ & $38[60.4 \%]$ & 63 \\
Total & $164[57.9 \%]$ & $119[42.1 \%]$ & 283 \\
\hline \multicolumn{4}{|c}{ Table 2. Results of Study } \\
\hline
\end{tabular}

(Adeno) Tonsillectomy was done in a total 241 patients while tonsillectomy alone was done in 16 patients and adenoidectomy alone was done in 32 patients.

Most common co-morbidity observed in the patients was obesity which was present in 65 cases out of $283(22.9 \%)$. Followed by otitis media with effusion (OME): 45 out of 283 cases (15.9\%) and then allergic rhinitis: 22 out of 283 cases (7.7\%). 139 cases out of 283 cases ( $49.1 \%$ ) were observed to have no co morbidities whatsoever. Other co morbidities are denoted in Table 3.

Concurrent procedure was performed in 45 patients with otitis media with effusion. Myringotomy with grommet insertion was done in 28 patients while myringotomy alone was done in 17 patients.

Post-operative complications were noted in 203 out of 283 cases (71.1\%) and have been denoted in Table 4. Out of 203, 76 patients $(26.8 \%)$ had post op fever which subsided within
24 hours of the procedure. 63 (22.2\%) patients showed inability to take oral feed within first 24 hours of the procedure. 47 (16.6\%) patients complained of pain which can also be the reason for delay in oral intake. Post-operative bleeding was found in 9 patients (3.1\%) out of which 6 stopped spontaneously with the formation of a small blood clot in the tonsillar fossa within 6 hours of surgery. 2 showed bleeding after 24 hours of surgery and were taken back to operation theatre for secondary ligation and cauterisation. 1 had post op bleeding on the 7 th day after surgery. And it stopped spontaneously without any active intervention. 6 patients complained of loosening of teeth following procedure and 2 patients had small lip laceration intraoperatively.

All the procedures gave satisfactory results and reported improvement of the patient's symptoms post operatively.

\begin{tabular}{|ccc|}
\hline Co-Morbidities & Number & $\mathbf{\%}$ \\
No co-morbidity & 139 & 49.1 \\
Obesity & 65 & 22.9 \\
OME & 45 & 15.9 \\
Allergic rhinitis & 22 & 7.7 \\
Asthma & 7 & 2.4 \\
Nasal polyp & 3 & 1.0 \\
Hyperinsulinism & 2 & 0.7 \\
\hline Table 3. Co-Morbidities Seen in the Patients \\
\hline
\end{tabular}

\begin{tabular}{|ccc|}
\hline Complications & No. of Cases & \% of Cases \\
Fever & 76 & 26.8 \\
Delay in food intake & 63 & 22.2 \\
Pain & 47 & 16.6 \\
Haemorrhage & 9 & 3.1 \\
Loosening of tooth & 6 & 2.1 \\
Lip laceration & 2 & 0.7 \\
\hline \multicolumn{2}{|c|}{ Table 4. Post-Operative Complications } \\
\hline
\end{tabular}

\section{DISCUSSION}

$\mathrm{T}$ \& A are common ENT procedures performed by otolaryngologists around the world. Every year, approximately $250,000 \mathrm{~T} \& \mathrm{~A}$ are performed in the US with the annual expenditure exceeding one and half billion dollars. ${ }^{6}$

In the recent few years, Mysore Medical College and Research Institute (MMCRI) has served as the tertiary health care institute in Mysore and approximately 350 - 400 T\&A have been performed each year in MMCRI. It has been observed that the number of T\&A being performed are going down most likely due to the increased use of antibiotics ultimately leading to decreased infection rates. 
In our study of 283 patient who underwent $\mathrm{T} \& \mathrm{~A}$ in the age group of 2 - 18 year, out of the two main indications, $57.9 \%$ were operated for obstruction and $42.1 \%$ were operated for infection. Our study states that obstruction is the major indication for the children up to 10 years of age and shows a decline as the child grows further. On the other hand, infection though less compared to the infection in patients of $4-10$ years of age was still the main Indication in the age group of 10 - 18 years as opposed to obstruction in that age group. These results compare favourably with that of Parker and Walner. ${ }^{7}$

Erickson et al did a 35-year epidemiological survey between 1970 and 2005 for 8106 patients which showed upper airway obstruction as an indication increased from 12 $\%$ to $77 \%$ during that period. Similarly, $90 \%$ of adeno tonsillectomies were performed for infections in 1970 which declined to $30 \%$ by 2005 . Similar results have been observed in our study as well and a study that was conducted in North America. ${ }^{8}$ These results can be attributed to the reduction in the size of adenoids with the increase in age of the child.

In this study, obesity is the most common co-morbidity that we encountered accounting for $22.9 \%$ of the total cases. This is comparable to the study done by Weerachai et al in Mahidol University which stated obesity as a co-morbidity in $17.9 \%$ cases over a 2-year study period. Childhood obesity is a serious rising public health issue which should be prevented as early in the age as possible. Childhood obesity shows strong associations with adulthood obesity and adverse health issues like OSAS. The prevalence of childhood obesity in our country is $19.3 \%{ }^{9}$ Childhood obesity is a fore runner of metabolic syndromes, poor physical health, mental disorders, respiratory problems and glucose intolerance, all of which can track in to adulthood.10 Obesity can be one of the factor associated with increase in tonsillar and adenoid size, ultimately leading to obstruction and OSAS in paediatric patients.

The $2^{\text {nd }}$ most common co-morbidity observed in our study was OME found in $15.9 \%$ of all cases as compared to $13.8 \%$ cases observed in the study by Weerachai et al. This can be due to recurrent infections of the eustachian tube following obstructive and infective adenoids and URTIs. Cases of concurrent OME were treated by doing myringotomy with or without grommet insertion.

All patients with and without co-morbidities showed improvement following T \& A. Significant improvement was seen in nocturnal enuresis and abnormal behaviour in the paediatric patients who presented with obstruction. These findings are comparable and are in agreement to studies done previously. ${ }^{11,12}$

Complications observed in our study were minor, detectable and easily treated. The most common complication observed was post-operative fever lasting for less than 24 hours was observed in 76 patients (26.8\%). Fever subsided within 24 hours to 48 hours without any sequelae. These stats were higher in our settings when compared to the study of Werrachai et al who reported it in $17.9 \%$ of their cases. 63 $(22.2 \%)$ patients refused to take orally in the $1^{\text {st }} 24$ hours which was observed majority in younger children. This led to their prolonged hospitalisation, more than 24 hours and few of the patients also complained of dehydration. ${ }^{13,14}$ All patients resumed adequate intake of oral feed within 48 hours of the procedure. This delay can also be attributed to post-operative pain in the throat which accounted for $16.6 \%$ cases in our series. Pain subsided within 24 hours of procedure after the patient was given one dose of intravenous paracetamol infusion followed by oral analgesics and antipyretic syrup. Haemorrhage was another complication seen in just 9 cases (3.1\%) in our study. Out of which 6 occurred in the first 6 hours of the procedure and no intervention was required for the same. Bleeding is generally a minor complication but can be a serious complication in smaller age group. 2 patients had bleeding after 24 hours and had to be taken up in operation theatre for active intervention and control of bleeding. Any loosened deciduous teeth should be noted preoperatively. Application of the mouth retractor should be performed very carefully to avoid loosening of teeth and any injury to lips and gums.

In our hospital setup, we generally keep the patients for overnight observation as opposed to the recent trends in the West. This can be attributed to comparatively cheaper expense as compared to the West and also the patients coming to our tertiary care centre are referred from far off primary healthcare centres.

Finally, as there are multiple studies which have used different study methods and surgical techniques, the strength of our results holds good for cold dissection and our experience in the southern Karnataka region over a period of one year of study. Therefore, the study should also be conducted for larger cohort and also for different methods of (Adeno) tonsillectomy.

\section{CONCLUSIONS}

(Adeno) tonsillectomy is a safe and effective procedure improving the quality of life of younger age group when performed for major indications. Obstruction is the major indication in younger age group which has been on the rise in recent few decades as opposed to infection which is the major indication in older age group children. Similar results were observed in our study. The most common co-morbidity seen in the literature was obesity observed in patients of OSAS and obstruction. Patients with co-morbidities should be kept for overnight observation in our opinion. Complications were easily detected and all patients recovered within 48 hours.

Data sharing statement provided by the authors is available with the full text of this article at jemds.com.

Financial or other competing interests: None.

Disclosure forms provided by the authors are available with the full text of this article at jemds.com.

\section{REFERENCES}

[1] Younis RT, Lazar RH. History and current practice of tonsillectomy. Laryngoscope 2002;112(8 Pt 2 Suppl 100):3-5.

[2] Manickavasagam J, Ali S, Quraishi MS. General practitioners' versus consultants' perspective on indications for paediatric tonsillectomy: current trends. J Laryngol Otol 2012;126(2):163-7.

[3] Gates GA, Folbre TW. Indications for adenotonsillectomy. Arch Otolaryngol Head Neck Surg 1986;112(5):501-2. 
[4] Silva S, Ouda M, Mathanakumara S, et al. Tonsillectomy underthreat: auditing the indications for performing tonsillectomy. J Laryngol Otol 2012;126(6):609-11.

[5] Sargi Z, Younis RT. Tonsillectomy and adenoidectomy techniques: past, present future. ORL J Otorhinolaryngol Relat Spec 2007;69(6):331-5.

[6] Paradise JL. Tonsillectomy and adenoidectomy. In: Bluestone CD, Stool SE, Alper CM, et al. eds. Pediatric Otolaryngology. $4^{\text {th }}$ edn. Philadelphia: Saunders 2003: p. 1210-22.

[7] Parker NP, Walner DL. Trends in the indications for pediatric tonsillectomy or adenotonsillectomy. Int J Pediatr Otorhinolaryngol 2011;75(2):282-5.

[8] Deutsch ES. Tonsillectomy and adenoidectomy. Changing indications. Pediatr Clin North Am 1996;43(6):1319-38.

[9] Ranjani H, Mehreen TS, Pradeepa R, et al. Epidemiology of childhood overweight \& obesity in India: a systematic review. Indian J Med Res 2016;143(2):160-74.
[10] Ahmed AO, Aliyu I, Kolo ES. Indications for tonsillectomy and adenoidectomy: our experience. Niger J Clin Pract 2014;17(1):90-4.

[11] Mora R, Salami A, Passali FM, et al. OSAS in children. Int J Pediatr Otorhinolaryngol 2003;67(Suppl 1):S229-31.

[12] Miman MC, Kirazli T, Ozyurek R. Doppler echocardiography in adeno tonsillarhypertrophy. Int J Pediatr Otorhinolaryngol 2000;54(1):21-6.

[13] Ross AT, Kazahaya K, Tom LWC. Revisiting outpatient tonsillectomy in young children. Otolaryngol Head Neck Surg 2003;128(3):326-31.

[14] Rothschild MA, Catalano P, Biller HF. Ambulatory pediatric tonsillectomy and the identification of high-risk subgroups. Otolaryngol Head Neck Surg 1994;110(2):203-10. 